\title{
The path to implementation of personalized medicine of aromatase inhibitors in patients with breast cancer
}

\author{
"Future personalized medicine with aromatase inhibitors is likely to \\ employ dynamic tumor phenotyping, molecular, pharmacogenomic \\ and epigenomic profiling and circulating tumor DNA monitoring."
}

First draft submitted: 17 August 2016; Accepted for publication: 17 August 2016; Published online: 28 October 2016

Keywords: aromatase inhibitors $\bullet$ clinical implementation $\bullet$ molecular profiling - personalized medicine $\bullet$ pharmacogenomics

Breast cancer is the most common cancer in women and the leading cause of cancer death, with over half a million deaths worldwide in 2012 [1]. The majority of breast cancers have estrogen (ER) and/or progesterone receptors $(\mathrm{PgR})$ and endocrine therapy is the backbone treatment for hormone-dependent breast cancer. The use of third-generation aromatase inhibitors (AIs), such as letrozole, anastrozole and exemestane, has significantly improved outcomes for postmenopausal women with ER positive $(\mathrm{ER}+)$ breast cancer. By selectively inhibiting aromatase in peripheral tissues, the third-generation AIs block the conversion of androgens to estrogen, producing a pronounced suppression of circulating estrogen levels. Despite the widespread use of AIs, not all patients with ER+ breast cancer benefit from AI therapy, and a marked interindividual variability in therapeutic efficacy and adverse events (AEs) has been reported. Response rates to AIs in advanced cancer vary from 32 to $70 \%$ [2], and many patients experience poor tolerance, with the main side effects being arthralgia, increased risk of osteoporosis and fractures [3]. Furthermore, in early breast cancer, $30-40 \%$ of ER+ tumors will develop acquired resistance to AIs, resulting in local or metastatic recurrence and decreased survival and quality of life $[2,4]$.

Personalized medicine is the tailoring and optimization of medical treatment to the characteristics of the individual patient and tumor. Given the variable clinical outcome and the potential for AEs and resistance, personalizing AI treatment has become a major objective in breast cancer. Therefore, there is an urgent need to identify biomarkers for the prediction of clinical efficacy and toxicity with the use of AIs.

\section{Current progress in personalized medicine}

ER status is currently the major molecular marker used routinely to guide clinical decision-making of the use of hormone therapy for women with breast cancer. However, a substantial proportion of breast cancer patients with ER+ tumors fail to respond to AI therapy. PgR, HER2 and Ki67 capture important aspects of breast tumor biology and are routinely measured. The presence of $\mathrm{PgR}$ has generally been regarded as an indicator of a functional ER pathway [5], HER2 positive (HER2 ${ }^{+}$) implies possible estrogenindependent tumor growth [6], and $\mathrm{Ki} 67$ is a marker of proliferation. The roles of these biomarkers in predicting response to treatment with AIs have been widely studied. However, the conclusions of these studies have been inconclusive, and these markers have only limited utility for predicting response to AIs [6].

The investigation of multigene signatures is a new strategy directed toward personaliz-
Xiaoman Liu

Faculty of Pharmacy, University of Sydney, Sydney, NSW 2006, Australia

Jane Beith

Chris O'Brien Lifehouse Centre, Sydney, NSW 2006, Australia

Siew-Kee Low

Faculty of Pharmacy, University of Sydney, Sydney, NSW 2006, Australia

Alan V Boddy

Author for correspondence:

Faculty of Pharmacy, University of Sydney, Sydney, NSW 2006, Australia alan.boddy@sydney.edu.au
Future
Medicine 
ing AI treatment. Oncotype Dx is one of the most validated multigene signatures, incorporating the expression profile of 21 genes associated with breast cancer. As a commercially available test, Oncotype Dx can predict recurrence risk in the adjuvant and neoadjuvant setting following treatment with AIs [7,8]. Early molecular changes after AI treatment may also help to predict AI response. By quantifying gene expression at baseline level and 2 weeks after neoadjuvant treatment with letrozole, a 205-gene signature could differentiate clinical responders and nonresponders [9]. Comparison of expression of genes related to immune signaling, proliferation, and apoptosis before and during letrozole therapy using a 4-gene signature also predicted response to AI treatment in the neoadjuvant setting with $96 \%$ accuracy. Moreover, in a validation data set, this model was found to be $91 \%$ accurate in patients treated with anastrozole [10]. However, in order to monitor the changes in gene expression, sequential biopsies are required. The integration of clinical parameters in a response prediction model may also be beneficial. Based on ER status, Ki67 levels, tumor size, nodal status and histological grade after treatment with neoadjuvant endocrine therapy, the preoperative endocrine prognostic index identified patients at low risk of relapse, thus avoiding unnecessary adjuvant chemotherapy [11].

\section{"...numerous biomarkers have been identified with potential to predict therapeutic efficacy and adverse events."}

In addition to investigating the molecular profiling of breast tumors, pharmacogenomic profiling of germline genetic variations may be important for personalizing AI therapy. Pharmacogenomics examines the influence of polymorphisms on pharmacokinetics, clinical response and AEs. The pharmacogenomics of AIs have been widely studied, with both candidate gene approaches and genome-wide association studies [12]. Genetic variants of the target aromatase gene (CYP19A1) itself have been extensively studied, and a number of $C Y P 19 A 1$ variants were found to associate with AI response, however not always consistently [12]. Polymorphisms may also associate with AI-related AEs. In the first genome-wide association study of AI pharmacogenomics, single nucleotide polymorphisms near the TCL1A gene, related to cytokine function, were identified as novel biomarkers of musculoskeletal AEs, thus providing new insight into the mechanism of this toxicity [13]. Genetic variants which affect the metabolism of AIs may account for the tenfold interpatient variability in plasma letrozole levels, with CYP2A6 genetic polymorphisms explaining $23 \%$ of the variation in letrozole concentrations [14]. This find- ing suggests that $C Y P 2 A 6$ polymorphisms may serve as biomarkers of efficacy.

Epigenetic modification, including histone modification, DNA methylation and noncoding RNAs, can regulate the expression of genes without change in the DNA sequence. Epigenetic signatures may have implications in AI resistance to breast cancer, as recently suggested. In a genome-wide study, specific chromatin-binding patterns of the repressive histone marker H3K27me3 were used to cluster good and poor prognosis patients into two groups, with prediction of patient outcome in a further independent set of AI-treated patients [15]. In another study, a microRNA microarray analysis identified overexpression of miR-125b in cellular models of acquired AI resistance, which was further proved to be associated with earlier relapse after endocrine therapy in breast cancer patients [16]. These biomarkers, if confirmed, may be used to stratify patients by predicted outcome. The epigenetic regulation of genes related to the pharmacokinetics of AI may also have a role in deciphering the individual variability in drug response and toxicities, with variation in methylation status has been reported to correlate with the expression of enzymes responsible for AI metabolism the liver $[17,18]$.

\section{Future perspective of the path toward personalized medicine}

Despite the promising studies described above, ER status is currently the only factor routinely used to select treatment with AIs for breast cancer patients. Biomarkers for molecular and pharmacogenomic profiling require further validation before implementation in routine clinical practice, and the development of epigenetic profiling may also help to identify biomarkers for personalized AI therapy. Discrepancies in research findings among different studies may be attributable to the heterogeneity in study designs, including differences in phenotype classification, treatment setting and population ethnicity. Moreover, adequate sample size is crucial to provide sufficient statistical power for the identification and validation of potential biomarkers. Therefore, standardized-design studies with adequate sample size facilitated by the contemporary analysis methods are required for further progress in biomarker implementation. The rapid development of genomewide microarray and next-generation sequencing technologies also provides the opportunity to identify novel genetic markers with no prior hypotheses. To take into account confounding factors, the clinical settings of the study must also be considered: as adjuvant treatment, response to AIs is confounded by tumor stage, metastatic spread of disease and inherent aggressiveness of the tumor and in the setting of advanced disease there 
may be multiple previous treatments. With fewer confounders, neoadjuvant therapy provides the best opportunity to identify tumor-related biomarkers of response.

The dynamic evolution of breast cancer necessitates sequential monitoring of the disease. Circulating tumor DNA (ctDNA) in the cell-free fraction of blood can provide a 'liquid biopsy', a surrogate for tumor biopsy, with tumor-specific sequence alterations. Analysis of ctDNA would facilitate personalized medicine by offering a noninvasive and innovative assessment of genotypic and phenotypic features of cancer, monitoring the progression of disease and tracking markers of resistance. The predictive and prognostic value of ctDNA in cancer has already been shown $[19,20]$, and investigation of ctDNA in AI therapy could provide an early assessment of treatment response or resistance [21].

Validation of clinical findings is crucial, as replication and functional studies verify and provide a mechanistic rationale. Prospective trials of validated biomarkers, followed by the pharmacoeconomic evaluation of cost-effectiveness, would confirm the role of Personalized medicine in decision-making in clinical practice. Clinical algorithms must be developed for interpretation and implementation of genetic data, and clinicians need to be educated about clinical genomics in order to apply the information. Moreover, treatment options for AIs include combinations with inhibitors of the PI3K/AKT/mTOR pathway or of CDK4/6, which have the potential to augment the benefit of existing AI

\section{References}

1 Ferlay J, Soerjomataram I, Dikshit R et al. Cancer incidence and mortality worldwide: sources, methods and major patterns in GLOBOCAN 2012. Int. J. Cancer 136(5), E359-E386 (2015).

2 Miller WR. Aromatase inhibitors: prediction of response and nature of resistance. Expert Opin. Pharmacother. 11(11), 1873-1887 (2010).

3 Gotay C, Dunn J. Adherence to long-term adjuvant hormonal therapy for breast cancer. Expert Rev. Pharmacoecon. Outcomes Res. 11(6), 709-715 (2011).

4 Dowsett M, Martin LA, Smith I, Johnston S. Mechanisms of resistance to aromatase inhibitors. J. Steroid Biochem. Mol. Biol. 95(1-5), 167-172 (2005).

5 Davies C, Godwin J, Gray R et al. Relevance of breast cancer hormone receptors and other factors to the efficacy of adjuvant tamoxifen: patient-level meta-analysis of randomised trials. Lancet 378(9793), 771-784 (2011).

6 Larionov A. Resistance to Aromatase Inhibitors in Breast Cancer. Alexey L (Ed.). Springer, CA, USA (2015).

7 Dowsett M, Cuzick J, Wale C et al. Prediction of risk of distant recurrence using the 21-gene recurrence score in node-negative and node-positive postmenopausal patients with breast cancer treated with anastrozole or tamoxifen: a TransATAC study. J. Clin. Oncol. 28(11), 1829-1834 (2010). therapy. Applying personalized medicine is, therefore, essential to ascertain optimal combination strategies.

\section{Conclusion}

With the aim of personalizing AI therapy, numerous biomarkers have been identified with potential to predict therapeutic efficacy and AEs. While some findings remain inconsistent, standardized designs and adequate sample size can be applied to minimize the heterogeneity among studies. Currently, biomarker research around AIs mainly focuses on molecular and pharmacogenomic profiling. Future personalized medicine with AIs is likely to employ dynamic tumor phenotyping, molecular, pharmacogenomic and epigenomic profiling and ctDNA monitoring. This will be facilitated by the growing availability of high-throughput and sensitive techniques, to provide clinical algorithms to achieve more specific patient stratification and more effective treatment.

\section{Financial \& competing interests disclosure}

The authors have no relevant affiliations or financial involvement with any organization or entity with a financial interest in or financial conflict with the subject matter or materials discussed in the manuscript. This includes employment, consultancies, honoraria, stock ownership or options, expert testimony, grants or patents received or pending, or royalties.

No writing assistance was utilized in the production of this manuscript.

Akashi-Tanaka S, Shimizu C, Ando M et al. 21-gene expression profile assay on core needle biopsies predicts responses to neoadjuvant endocrine therapy in breast cancer patients. Breast 18(3), 171-174 (2009).

9 Miller WR, Larionov A, Renshaw L et al. Gene expression profiles differentiating between breast cancers clinically responsive or resistant to letrozole. J. Clin. Oncol. 27(9), 1382-1387 (2009).

10 Turnbull AK, Arthur LM, Renshaw L et al. Accurate prediction and validation of response to endocrine therapy in breast cancer. J. Clin. Oncol. 33(20), 2270-2278 (2015).

11 Ellis MJ, Tao Y, Luo J et al. Outcome prediction for estrogen receptor-positive breast cancer based on postneoadjuvant endocrine therapy tumor characteristics. J. Natl Cancer Inst. 100(19), 1380-1388 (2008).

12 Liu X, Low SK, Boddy AV. The implications of genetic variation for the pharmacokinetics and pharmacodynamics of aromatase inhibitors. Expert Opin. Drug Metab. Toxicol. 12(8), 851-863 (2016).

13 Ingle JN, Schaid DJ, Goss PE et al. Genome-wide associations and functional genomic studies of musculoskeletal adverse events in women receiving aromatase inhibitors. J. Clin. Oncol. 28(31), 4674-4682 (2010).

14 Desta Z, Kreutz Y, Nguyen AT et al. Plasma letrozole concentrations in postmenopausal women with breast cancer 
are associated with CYP2A6 genetic variants, body mass index, and age. Clin. Pharmacol. Ther. 90(5), 693-700 (2011).

15 Jansen MP, Knijnenburg T, Reijm EA et al. Hallmarks of aromatase inhibitor drug resistance revealed by epigenetic profiling in breast cancer. Cancer Res. 73(22), 6632-6641 (2013).

16 Vilquin P, Donini CF, Villedieu M et al. MicroRNA-125b upregulation confers aromatase inhibitor resistance and is a novel marker of poor prognosis in breast cancer. Breast Cancer Res. 17, 13 (2015).

17 Hammons GJ, Yan-Sanders Y, Jin B, Blann E, Kadlubar FF, Lyn-Cook BD. Specific site methylation in the 5'-flanking region of CYP1A2 interindividual differences in human livers. Life Sci. 69(7), 839-845 (2001).
18 Kacevska M, Ivanov M, Wyss A et al. DNA methylation dynamics in the hepatic CYP3A4 gene promoter. Biochimie 94(11), 2338-2344 (2012).

19 Diaz LA Jr, Bardelli A. Liquid biopsies: genotyping circulating tumor DNA. J. Clin. Oncol. 32(6), 579-586 (2014).

20 Krebs MG, Hou JM, Ward TH, Blackhall FH, Dive C. Circulating tumour cells: their utility in cancer management and predicting outcomes. Ther. Adv. Med. Oncol. 2(6), 351-365 (2010).

21 Schiavon G, Hrebien S, Garcia-Murillas I et al. Analysis of ESR1 mutation in circulating tumor DNA demonstrates evolution during therapy for metastatic breast cancer. Sci. Transl. Med. 7(313), 313ra182 (2015). 